\title{
POLÍTICAS PENITENCIARIAS Y
} EXPERTOS EN LA PROVINCIA DE BUENOS AIRES EN LA DÉCADA DEL '30: DIAGNÓSTICOS, PROPUESTAS Y TRANSFORMACIONES DEL RÉGIMEN CARCELARIO

\section{Ignacio Araujo*}

Universidad Nacional de General Sarmiento

$\triangle$ ignacioaraujo.8@gmail.com

Recibido: 23 de agosto de 2020 Aceptado: 30 de noviembre de 2020 DOI: $10.46553 /$ colec.32.1.2021.p129-157

Resumen: Este artículo indaga las propuestas y transformaciones sobre el régimen carcelario que tuvieron lugar en la provincia de Buenos Aires durante la década del 30 . Analiza el papel de los expertos en conocer la situación carcelaria provincial y las medidas sugeridas a partir de estos diagnósticos. En este sentido demostraremos que existió una preocupación de los funcionarios provinciales, el rol de expertos con amplia trayectoria académica y gubernamental en el estudio de la situación carcelaria provincial, y cómo esas propuestas informaron las políticas tendientes a la transformación del sistema penitenciario. Así planteado este trabajo demuestra los vínculos entre políticos y expertos, así como restituye el dinamismo que cobró la cuestión carcelaria en los años 30 en la Provincia de Buenos Aires.

Palabras claves: expertos; cárceles; Estado; Provincia de Buenos Aires

\footnotetext{
* Profesor Universitario en Educación Superior en Historia (UNGS) y Maestrando en Ciencias Sociales (UNGS-IDES). Esta investigación se realizó gracias a una beca de investigación de la UNGS bajo la dirección de Jeremías Silva. Agradezco sus sugerencias y comentarios.
} 


\begin{abstract}
This paper explores the proposals and changes of the prison regime that took place in Buenos Aires Province during the 30s. It analyzes the role of the experts in knowing the provincial prison situation and the suggested measures based on these diagnoses. Thus, we will show that there was a concern to the province officials, the role of experts with wide academic and governmental trajectory in the study of the provincial prison situation, and how these proposals reported the policies tending to change the prison system. Presented in this way, this article demonstrates the connection between politicians and experts, and also proves how dynamic the prison issues were during the 30's in Buenos Aires Province.
\end{abstract}

Keywords: Experts; Prisons; State; Buenos Aires Province

\title{
I. Introducción
}

Al asumir como gobernador de la Provincia de Buenos Aires, Federico Lorenzo Martínez de Hoz reconocía en 1932 que "el problema carcelario, tan estudiado y discutido, no ha sido resuelto" (Ministerio de Gobierno 1933, 169). Para el gobernador resultaba evidente que la situación penitenciaria provincial acarreaba múltiples déficits entre los que señalaba: la falta de edificios, la imposibilidad de garantizar un régimen de trabajo y las dificultades de hacer cumplir el Código Penal en ese contexto. Agregaba que, si bien la situación financiera era crítica y no permitía "desarrollar iniciativas como sería la de construcciones de cárceles, que requieren la inversión de grandes capitales", era imperioso atender tal situación y ofrecer soluciones. De allí que una de sus primeras medidas fuese la conformación de una comisión de expertos para atender al "problema carcelario".

Como han señalado con agudeza Ricardo Salvatore y Carlos Aguirre (1996) en los ciclos de reformas penitenciarias de América Latina podían ubicarse fases de entusiasmo, descreimiento e interés renovado. Justamente, en este trabajo argumentaremos que la década de 1930, bajo el dominio de las gestiones conservadoras, constituyó un momento central en la reforma del sistema penitenciario provincial. Tal como lo exhibe la cita del gobernador en 1932 con la que abrimos este trabajo los funcionarios provinciales reconocieron la necesidad de atender a la situación carcelaria, 
y en consecuencia, encargaron a expertos con amplia trayectoria académica y gubernamental el estudio de la situación carcelaria provincial. Como buscaremos demostrar, fue sobre la base de las propuestas expertas que las autoridades impulsaron políticas tendientes a la transformación del sistema penitenciario. Así planteado, este artículo se propone iluminar la centralidad de esas preocupaciones en ámbitos políticos, los resultados que diseñaron los expertos y cómo estos informaron la planificación de políticas provinciales. En particular pondremos el foco en el gobierno de Federico Martínez de Hoz (1932-1935) y de Manuel Antonio Fresco (1936-1940).

En los últimos años, la historia de la prisión en Argentina ha sido objeto de nuevas lecturas, revisitando clásicos postulados y abogando por una perspectiva que tenga en cuenta no solo la multiplicidad de actores que conforman los espacios carcelarios sino también el devenir de prácticas y trayectorias a lo largo de distintas etapas temporales. Los primeros estudios sobre la conformación de los sistemas penitenciarios han demostrado de manera notoria la importancia que tuvo para las elites gobernantes, a fines de siglo XIX, el desarrollo de un "castigo civilizado" (Caimari 2004; Barreneche y Salvatore 2013). Así mismo también se ha ponderado el papel que jugó la criminología positivista dentro de las burocracias estatales no solo en Argentina sino en Latinoamérica a partir de una fluida circulación de saberes y de conceptos devenidos en proyectos de políticas gubernamentales (Salvatore 2001; Silva 2013; León León 2014). De igual manera, investigaciones recientes ponen el foco en la constitución de sistemas carcelarios provinciales: esta creciente literatura se apoya en una reducción de escalas demostrando que existieron realidades propias de la periferia que escapan a las miradas que han centrado y conformado visiones totalizadoras en base a los establecimientos nacionales de la Capital Federal o Ushuaia. De esta manera los casos provinciales son relevantes ya que, por un lado, generan desafíos metodológicos y estrategias renovadoras en el campo historiográfico como en el caso rosarino (Piazzi 2012); y por otro lado, enmarcan procesos de reforma que están delimitados por otras temporalidades como en Córdoba o La Pampa durante la primera parte del siglo XX o la cárcel-cuartel y las reformas en materia reglamentaria en Tucumán (González Alvo 2013 y 2015; Luciano 2015; Flores 2015). No obstante, la experiencia penitenciaria de la provincia de Buenos Aires, que poseía el mayor número de penados del país ha sido poco atendida. 
Recientemente múltiples investigaciones han comenzado a subsanar este "vacío" historiográfico desde la génesis del sistema penitenciario bonaerense hasta su convivencia con instituciones religiones de renombre (Yangilevich 2017; Giacomelli 2018; Belzunces 2020). No es casual, que los numerosos trabajos sobre este periodo central en la construcción estatal del castigo moderno contrasten con la menor atención que recibieron otros momentos de reformas, sobre todo en el siglo $\mathrm{XX}$, pero que es necesario indagar para poder tener una perspectiva histórica sobre los desarrollos penitenciarios actuales en un momento en el que se discute el rol y la eficiencia de los espacios carcelarios.

De la misma forma, las investigaciones sobre las agencias estatales y sus vínculos con las elites técnicas también han tenido un notable desarrollo. Múltiples estudios han indagado la administración a nivel nacional desde la conformación del Estado argentino demostrando el proceso de delimitación de sus áreas de intervención. Dichas intervenciones no se encontraban preestablecidas en el engranaje estatal, sino que se fueron entretejiendo en un camino marcado por la multiplicidad de actores y prácticas especializadas (Lobato y Suriano 2014). Por ende, los trabajos de este campo han iluminado las relaciones entre los contextos políticos, las racionalidades burocráticas y los saberes expertos para comprender la constitución, puesta en práctica y devenir de diferentes organismos gubernamentales (Bohoslavsky y Soprano 2010). En relación a los expertos, la literatura ha demostrado como los técnicos han tenido un papel importante en la delineación de políticas públicas: sujetos que se insertan en el Estado con la potestad de diagnosticar los problemas sociales y que a la vez fueron fuente de poder político para las gestiones gobernantes (Palermo 2006). En ese proceso de inserción en el Estado se adquiere una transformación de ese saber cómo operativo, que es demandado y a la vez constitutivo del Estado Moderno (Plotkin y Zimmermann 2012, 10). La preocupación por las escalas de análisis, también en este caso, llevó a los historiadores a descentrar el marco espacial de análisis de las transformaciones estatales, recuperando las especificidades de las provincias y territorios nacionales y demostrando que lo ocurrido en la capital del territorio no podía extrapolarse a todo el país (Ortiz Bergia 2015; Di Liscia y Soprano, 2017). Los desarrollos acontecidos dan cuenta de las novedades que arroja una reducción en la escala de análisis durante el siglo 
XX y que invita a pensar otras temporalidades y otras relaciones entre distintos registros, así como las tensiones entre el gobierno nacional y provincial (Béjar 2005, Biernat y Ramacciotti 2016; Raffa 2018). En sintonía con estos estudios, los desarrollos estatales de la provincia de Buenos Aires han merecido un creciente interés en las últimas décadas. Dejar de dar por sentada la imbricación entre las historias de la provincia y de la nación ha permitido problematizar los conflictos generados en torno al papel que debía desempeñar en la unificación nacional esta verdadera "primus inter pares" que fue Buenos Aires. No sorprende por ello que, tal como indica Ternavasio (2013), la preparación de los estudios sobre este territorio implicará la necesaria puesta en cuestión de los lugares comunes que han marcado la historiografía de la "primer provincia argentina": el unitarismo o centralismo, la estancia ganadera y la homologación entre clase terrateniente y dirigencia política. Dejando de lado estos puntos que hacen a la temática aplicada a la formación del estado nacional, también los estudios acerca de la provincia se han desplegado a través de las fuentes documentales que ella provee. Es de esta manera, que diversos historiadores centraron sus investigaciones demostrando las especificidades en las prácticas, la sociabilidad, las burocracias y los fenómenos económicos y políticos del escenario bonaerense (Barreneche 2010; Fernández 2014; Béjar 2005; Palacio 2013).

Así concebido, este artículo aspira a dialogar con estas dos producciones historiográficas poniendo el foco en las propuestas de reforma y en las transformaciones que acontecieron en las cárceles de la Provincia de Buenos Aires durante la década del 30. En la primera parte nos concentraremos indagando la situación penitenciaria para 1932, a partir del informe realizado por expertos a pedido del Poder Ejecutivo Provincial, dando cuenta de los diagnósticos y las propuestas de reforma de los especialistas. En la segunda parte nos detendremos en las transformaciones institucionales a partir del análisis de la legislación y de las innovaciones en la estructura burocrática del Estado provincial durante las gestiones de Martínez de Hoz y de Manuel Antonio Fresco, ponderando la relación entre dichas reformas y los saberes expertos. La base documental sobre la que se desarrollará esta investigación está conformada por fuentes oficiales de diversa índole: informes oficiales, legislación provincial, memorias de gobierno y revistas especializadas de carácter penitenciario. 


\section{Panorama carcelario. Expertos y diagnósticos}

Para principios de la década del 30 el panorama a nivel nacional, y sobre todo a nivel provincial, se mostraba convulsionado en primera instancia por la interrupción democrática que cambió el juego político a nivel nacional y provincial, y en segunda instancia, pero no menos importante, la crisis del 29 que definió un escenario económico complejo para las provincias. Hasta 1934-1935 la economía bonaerense sufrió las consecuencias de la crisis que provocó la caída del comercio internacional en detrimento de los productos primarios, situación que retrajo su producción ganadera (Degregori 1996, 32). Asimismo, se sumaba la inestabilidad fiscal y el pago de los servicios de la deuda que provocaron, entre otras cosas, rebajas de salarios, reducción del empleo público y suspensión de jubilaciones (Béjar, 2005, 113-114). En este contexto la situación carcelaria atravesó dos procesos paralelos muy en común a otros ámbitos estatales: la falta de un sólido presupuesto y la ausencia de políticas carcelarias que tengan como horizonte transformar el complejo escenario en que se encontraban hasta el momento. Recordemos que el sistema carcelario bonaerense se consolidó reglamentariamente a fines del siglo XIX y presentó desde un primer momento la característica de no poseer los mismos éxitos a nivel nacional como el Presidio de Ushuaia o la Penitenciaria Nacional, entendidas en ese momento como "cárceles modelo". Es preciso recordar que, con la federalización de la Capital, la provincia perdió la Penitenciaría, devenida en nacional, sufriendo las consecuencias de tal situación política (Caimari 2004). Cómo demostró Melina Yangilevich (2017) la provincia, desde principios de siglo XX, presentó los mismos "pantanos punitivos"1 que los territorios nacionales y provincias del interior que no podían hacer frente a las demandas de higiene e infraestructura. Para el año 1887 se sancionó el Código Penal de la Nación que no tuvo efectos positivos o de mejora en los establecimientos, no siempre adecuados a los requerimientos del momento (Silva 2013). En general las cárceles bonaerenses fueron construidas durante ese período destacando el presidio de Sierra Chica como el establecimiento que albergaba mayor cantidad de presos. Inaugurado en Olavarría en 1881 tuvo desde un primer momento problemas para concluir sus obras de

${ }^{1}$ Ver Caimari (2002). 
infraestructura y forjarse como una institución consolidada. Los esfuerzos a principios de siglo XX por torcer la situación carcelaria significó el desarrollo de iniciativas tendientes a una mayor organización. En este marco, en 1911 se creó la Inspección General de Prisiones que se encargó de la administración de las cárceles en relación a los suministros y la reglamentación carcelaria pero que funcionaba supeditada a dos entes en simultáneo ya que las cárceles estaban divididas entre el Poder Ejecutivo provincial y el Judicial. ${ }^{2}$ Los establecimientos que dependían del Ejecutivo eran: el penal de La Plata, la Penitenciaria y Cárcel de Mujeres y el penal de Sierra Chica (además del Departamento de Policía y la Cárcel de Detenidos de la calle 14 de La Plata). Por otro lado, la Suprema Corte de Justicia tenía la potestad de las cárceles departamentales de La Plata, Mercedes, San Nicolás, Dolores, Bahía Blanca así como de la cárcel creada en 1933 en la localidad de Azul. No obstante, la cantera de la Cárcel de Sierra Chica funcionaba bajo el régimen del Ministerio de Obras Públicas.

Sí las preocupaciones por las condiciones y el régimen carcelario siguieron presentes en las primeras décadas del siglo XX, recién en los años 30 se tomaron medidas políticas concretas para atender a esta situación. Justamente, el 3 de octubre de 1932 el Poder Ejecutivo de la Provincia de Buenos Aires, a cargo del gobernador Federico Lorenzo Martínez de Hoz, estableció a partir del Decreto $\mathrm{N}^{\circ} 140$ la conformación de la "Comisión de Estudio del Régimen e Institutos carcelarios de la Provincia de Buenos Aires" (Paz Anchorena 1934). Dicha comisión buscaba estudiar el régimen penitenciario y llevar a cabo una propuesta de reforma que permitiese reorganizar las cárceles provinciales, tal como reclamaban expertos y funcionarios. Esto implicaba adecuar el sistema penitenciario a las premisas básicas que debían cumplir las instituciones de castigo: trabajo, instrucción y reforma de los penados. El objetivo que impulsaba al Poder Ejecutivo Provincial (PEP) a convocar a una comisión de expertos, no solo buscaba una mejor organización para la readaptación social de los penados sino

\footnotetext{
${ }^{2}$ La ley fue sancionada el 5 de septiembre de 1910 y paso a funcionar como una agencia dependiente del Ministerio de Gobierno, más precisamente de la Dirección de Suministros. Estaba conformada por un Inspector General, dos inspectores de menor rango acompañados por dos escribientes (Orden del Día 6362, Jefatura de la Provincia de Buenos Aires, 1910).
} 
también en delimitar las atribuciones estatales bonaerenses en lo relativo a los establecimientos. Esto provocó problemas con los altos rangos de la corte que veían anti constitucional la medida por parte del Ejecutivo y que tuvo su respuesta en octubre de ese año por parte del juez Dr. Casaux (Ministerio de Gobierno 1933). El hecho se originaba en que la Corte Suprema de Justicia estaba a cargo, como hemos dicho anteriormente, de las cárceles de encausados (aquellos sujetos que están atravesando procesos judiciales y por lo tanto no tenían condena firme), mientras que las cárceles de condenados se encontraban bajo la órbita de atribuciones del gobernador. El Ejecutivo respondía trayendo a colación este problema a partir de un decreto del PEP citando al ex-ministro de gobierno provincial de 1913, Francisco Uriburu, quien señalaba que:

Todo aquello que divida en dos fases este régimen no tendrá inconvenientes insalvables, eso lo creo, pero sí, puede tener graves inconvenientes; porque si es verdad que la aspiración que puede tener el gobierno es que los pocos condenados que están en las cárceles de detenidos y encausados pasen a la jurisdicción que les corresponde, es decir, a la jurisdicción del Gobierno, es indudable también que ese régimen interno de las cárceles, para ser eficaz tiene que dominar todo el cuadro carcelario, tanto de detenidos, como de condenados. (Paz Anchorena 1934, 12)

E1 PEP en su justificación fundamentaba que, aunque la Corte Suprema Provincial (CSP) pudiese ejercer su jurisdicción en el régimen interno de las cárceles de detenidos, todo lo relativo a la organización y reforma de los penados estaba establecido en el presupuesto provincial y, por tanto, supeditado al Poder Legislativo. Por lo tanto, Martínez De Hoz junto al aval de su gabinete y de los legisladores dejó sin efecto las acusaciones y alegatos del poder judicial, sosteniendo la conformación de la comisión de estudio. Ésta, marcó el punto de partida para poder llevar a cabo un relevamiento minucioso del sistema carcelario provincial, que se había limitado a las inspecciones y a fallidos intentos de llevar a cabo su estudio en diferentes ocasiones (Paz Anchorena 1934, 13). Por ende, la conformación de esta Comisión revela el compromiso político con la reforma de las prisiones, siempre declamada pero no concretada. La presidió el abogado y profesor 
de la Facultad de Derecho de la Universidad de Buenos Aires, Dr. José María Paz Anchorena ${ }^{3}$, y la integraban el asesor de gobierno, Dr. Juan Silva Riestra $^{4}$; el Director General de Higiene de la Provincia, Atilio Viale, y el secretario asignado ad honorem, César Etcheverry.

De esta forma, luego de llevar a cabo un minucioso trabajo, el 20 de abril de 1934 el Dr. Paz Anchorena elevó el informe al gobernador Martínez de Hoz, detallando toda la organización penitenciaria y proponía los medios que se debían llevar a cabo para la solución de los problemas que presentaba el régimen carcelario. El informe comenzaba retomando iniciativas previas de análisis de la situación carcelaria, como por ejemplo la sanción en 1914 de la Ley 3544 en la cual la legislatura provincial establecía la creación de una Comisión de Cárceles, que sin embargo quedó sin efecto al poco tiempo. La ley atribuía a esta comisión ciertos principios con los que coincidía en torno a la construcción de cárceles, ampliación, higienización y adopción de "medidas tendientes a la moralización y corrección de los recluidos en las mismas" (Ministerio de Gobierno 1914). Por lo tanto, Paz Anchorena remarcaba esta experiencia infructuosa para ponderar los esfuerzos realizados bajo la gestión conservadora de Martínez de Hoz en relación a otras gestiones anteriores. De allí, la primera cuestión en la que se detenía el informe era en describir la situación de los establecimientos en la provincia. Hasta ese momento la Provincia contaba con doce establecimientos carcelarios. El informe destacaba la superpoblación que existía en los establecimientos señalando que en las cárceles para los condenados tenían una capacidad para 866 personas y alojaban a 1253 , presentando la situación más crítica el Presidio y Penitenciaría de Sierra Chica que contaba con 536 plazas y albergaba a 836 penados. Las cárceles de encausados tampoco arrojaban un panorama alentador, teniendo espacio para 1750 personas, todos los establecimientos presentaban sobrepoblación

\footnotetext{
3 Se recibió de abogado en 1917 con la tesis "La prevención de la delincuencia", distinguida con Diploma de Honor. Se destacó por su actuación como representante oficial en ámbitos internacionales especializados, como lo hizo en el IX Congreso Penitenciario Internacional de Londres en 1925 (Silva 2019).

4 “Desempeñó las funciones de Asesor de Gobierno, Interventor en el Departamento del Trabajo y Fiscal del Estado, en los años 1932, 1933 y 1934. Presidente de la Sociedad Argentina de Criminología. Jurisconsulto y Profesor universitario en la UBA" (Asesoría General de Gobierno, Web PBA 2020).
} 
arrojando un número total de alojados de 1982 (Paz Anchorena 1934, 7191).

Asimismo, el informe se detenía en tres problemas comunes a todos estos establecimientos. El primero era las condiciones de higiene, caracterizada como precaria desde todo punto de vista, en particular en relación a dos cuestiones: en lo concerniente a los establecimientos, así como a los propios detenidos. Los edificios databan en su mayoría de fines del siglo XIX y no contaban con remodelaciones posteriores encontrándose deteriorados, una situación que no distaba de otros establecimientos carcelarios provinciales y nacionales (Bohoslavsky y Casullo 2003; Caimari 2004; Silva 2013; González Alvo 2015; Luciano 2015). Al mismo tiempo los baños dependían en muchos casos de la propia voluntad de los presos de mantenerlos, y no contaban con los servicios imprescindibles, por ejemplo, el agua caliente o instrumentos para el aseo personal que llevaban a que la población carcelaria se encontrara en condiciones antihigiénicas, con barbas o melenas largas "que contribuyen a hacer más repulsivo el cuadro carcelario que estamos describiendo" (Paz Anchorena 1934, 27). El segundo problema refería a la cuestión del trabajo, definido como insuficiente ya que gran parte de la población carcelaria no podía acceder a realizar tareas que para los expertos era la base de la reeducación de los penados. Esto puede ilustrarse en el caso del Penal de Sierra Chica pues tan solo trabajaban 431 personas de las 836 que concentraba este penal, dejando como saldo 405 condenados desocupados. De igual modo, en algunos casos el trabajo consistía en el simple mantenimiento del establecimiento o en talleres de escasos recursos por la falta de equipamiento apropiado.

El último punto del informe hacía referencia a la falta de instrucción. Esta no era solo entendida en lo concerniente a garantizar la educación de los penados que la necesitasen, sino también a la enseñanza religiosa y moral. Sobre esta situación, el informe enfatizaba la falta de recursos económicos para contratar maestros y profesores que dieran las clases, lo que perjudicaba uno de los principales propósitos del sistema carcelario: la reforma del penado. A la falta de instrucción básica, se sumaba la falta de enseñanza religiosa en los establecimientos, ya que para los autores del proyecto la reforma del sujeto era concebida de manera integral y debía incluir la educación religiosa. A decir verdad, este cuadro de situación descrito no distaba de otras realidades carcelarias a lo largo de todo el 
territorio nacional denunciadas por diferentes sectores desde principios de siglo XX.

Pero el informe no sólo ofrecía un diagnóstico sombrío, también proponía medidas concretas a fin de mejorar la situación carcelaria provincial. La primera propuesta estipulaba la modificación y readaptación de las estructuras edilicias para poder saldar el grave problema de la superpoblación, y a la vez dotar a los establecimientos de un sentido práctico y científico para el tratamiento del penado. Se debía en algunos casos construir pabellones o ampliarlos, como en el caso de las cárceles de encausados de San Nicolás o la de Bahía Blanca, o en casos extremos el desalojo total y la construcción de un nuevo edificio para terminar con la cuestión de la superpoblación como en el caso de la Penitenciaria de La Plata. Los autores del informe señalaban que en éste último caso el Estado debía edificar una gran cárcel de encausados con capacidad para mil procesados y que podía realizarlo en los terrenos fiscales próximos. En cuanto al tratamiento del penado y el mejoramiento edilicio el informe era contundente: debía asegurarse un hombre por celda. Se concebía que era una condición central, pues sostenían que: “el estado debe hacer desaparecer lo antes posible el sistema de dos hombres por celda cuya perniciosidad queda de manifiesto solo con exponer una de sus fatales consecuencias: la homosexualidad" (Paz Anchorena 1934, 34).

La segunda propuesta se detenía en los reglamentos internos de las cárceles. En algunos establecimientos carecían totalmente de uno, o se regían por viejos reglamentos del siglo XIX. La comisión recomendaba que el Consejo Carcelario de la provincia debía abocarse de inmediato al estudio y redacción de reglamentos para condenados y procesados. Esto era de suma importancia para la buena administración, la disciplina y el orden que buscaban promover en todo el sistema carcelario. Los autores señalaban que los reglamentos eran la carta magna de los reclusos, donde debían estar sus deberes y especificarse detalladamente todas las obligaciones con sus respectivas sanciones en caso de incumplimiento, sin dejar de lado los premios para los penados que tenían un buen comportamiento.

La tercera propuesta estaba relacionada con un tema que promovió sucesivos debates en todos los órdenes estatales: el régimen de trabajo. Ante esto, el informe era determinante: "el régimen del trabajo en las cárceles es el escollo donde tropiezan todas las organizaciones carcelarias" (Paz 
Anchorena 1934, 41). Puede indicarse que en general las cárceles provinciales no se adecuaban hasta ese momento ni las aspiraciones de la doctrina positivista respecto a los beneficios del trabajo, a la que adherían los miembros de la comisión, ni las necesidades utilitarias que es uno de los fines que se persigue con su implantación. El Estado había fracasado en la organización de los talleres carcelarios ya sean por carecer de personal técnico encargado de dirigirlos o por la ineficacia del personal obrero que representa el recluso. Esta doble incapacidad que ilumina el informe traía como consecuencia un costo desproporcionado del mantenimiento de dichos talleres y el bajo provecho para la regeneración de los penados. El costo hasta ese periodo era categórico, la provincia gastaba (en moneda nacional): \$2.060.141. Al mismo tiempo los pocos talleres con los que se contaba enseñaban oficios inútiles que no generaban en los reclusos la capacidad para poder llevarlos a cabo al cumplir la condena. Los convertía en obreros de "fácil aprendizaje", contrariando la aspiración de proporcionarles un recurso adecuado para la liberación y un medio en sus términos, moralmente aceptable, para ganarse la vida.

Precisamente esto despertaba en la comisión la obligación de proponer al gobierno provincial la idea de abandonar estas prácticas que carecían de eficiencia y que no arrojaban a grandes resultados cuantitativos ni cualitativos. Para eso se formulaba una idea que podíabrindar solución a los dos problemas de falta de espacio e instrucción: la implantación de un sistema de trabajo al aire libre, fuera de los establecimientos. La comisión explayaba su fundamentación a partir del estudio de antecedentes a nivel internacional con una vasta bibliografía. Si bien esta cuestión era debatida a nivel mundial como en el Congreso Penitenciario Internacional celebrado en Budapest en 1905, en el período de entreguerras continuaba siendo objeto de acaloradas discusiones como en el Primer Congreso Penitenciario de Derecho Penal de 1926 en la cual se presentaron distintos estudios sobre la implementación de este tipo de trabajo. El trabajo al aire libre ya había sido exitosamente establecido en Grecia, descartando las objeciones derivadas de su dificultad de vigilancia, y que tenía como factor positivo el bajo costo (la provincia contaba con extensas tierras fiscales), y no requería de instalaciones especiales (Paz Anchorena 1934, 48). Del mismo modo, los autores del informe citaban sobre esta cuestión el trabajo presentado por los doctores Juan Ramos y Jorge Coll en el Congreso de 1926 donde 
enfatizaban la "ventaja" que tenía el obrero argentino, en términos de una raza diferente capaz de soportar cualquier tipo de penuria como la vida en carpa, y las altas y bajas temperaturas. La comisión por esto último declara efusivamente:

Imitemos este sistema; considérense a nuestros condenados como obreros de un gran establecimiento agrícola, sin el aspecto tétrico de una cárcel, sin los murallones que limitan el horizonte, sin corromper los pulmones, y la Provincia habrá dado un gran paso por el bien de sus condenados y sus arcas fiscales. (Paz Anchorena 1934, 53)

La cuarta propuesta se detenía en las soluciones que debían brindarse en salubridad e higiene. Los autores del informe creían que era necesario que el servicio médico sea reorganizado, promoviendo la visita de un médico que realice una historia clínica de los penados a fin de que se indiquen las normas generales que deben someterse, en este caso, a los enfermos. En lo que concierna a las condiciones de los baños resultaba indispensable mejorarlos con simples medidas reglamentarias e instalaciones de bajo costo. Para Paz Anchorena y los demás integrantes de la comisión existían formas simples de conciliar la higiene con el aspecto físico y el respecto por la dignidad humana.

La quinta y última propuesta, se concentraba en proponer soluciones respecto dela instrucción educativa y religiosa, ya altamente cuestionada e informada por su falta de solidez en el tiempo. Era menester dotar cuanto antes de maestros a los establecimientos, y que la enseñanza se reduzca solo a la elemental, contemplando la lectoescritura y la solución de algunas operaciones aritméticas fundamentales. Asimismo, se fomentaba la instalación de bibliotecas en las cárceles, que hasta ese momento se encontraban en un estado deplorable o de ausencia como, por ejemplo, la cárcel de Dolores. Por lo tanto, resultaba imperioso que el gobierno promoviera este tipo de bibliotecas y proveyera a los establecimientos de los maestros necesarios ya que esto redundaría sin dudas, para la comisión, en un beneficio directo para el recluso y la sociedad. En este sentido, es indudable la influencia de la religión como elemento educador, que sirviese a la vez como vehículo de determinas ideas morales, siendo en consecuencia un deber del Estado remediar la debilidad moral del recluso. 
De todo lo anterior sucintamente expuesto sobre el diagnostico se desprende a grosso modo que la solución del problema carcelario en la provincia, en palabras de la comisión, no ofrecía grandes dificultades, pero sí llegaban a ser múltiples y sumamente variadas. Los autores remarcaban la necesidad y beneficios de "unidad de dirección", una centralización que se debía llevar a cabo para poder reorganizar de manera eficiente el sistema carcelario teniendo en cuenta los distintos ítems que se desarrollaron, en tanto representan una enunciación concreta de soluciones de fácil alcance, siempre que existiesen oportunas medidas legislativas y administrativas. Por ende, la premisa de la Comisión consistía en uniformar el sistema, establecer parámetros comunes y adecuar el régimen a las discusiones que desde principios de siglo estaban llevándose a cabo en ámbitos expertos. Estas propuestas no distaban de las premisas de la criminología positivista que compartían numerosos expertos en derecho y especialistas carcelarios. Como ha demostrado Ricardo Salvatore (2001) las principales premisas de la criminología positivista eran la educación, el trabajo y disciplina. De la misma manera, la propuesta de crear un organismo que centralice la gestión penitenciaria estaba en consonancia con las transformaciones sustantivas que se producían en el Estado Nacional que en 1933 conformó dentro del Ministerio de Justicia e Instrucción Pública la Dirección General de Institutos Penales (Silva 2013).

\section{Transformaciones institucionales}

Como lo anticipamos el informe contenía un diagnóstico detallado de la situación carcelaria bonaerense. Del mismo modo, ofrecía una serie de medidas concretas a poner en práctica para dar solución a problemas que arrastraban los establecimientos de castigo, en muchos casos, desde fines del siglo XIX. Asimismo, la convocatoria a la comisión revelaba que las distintas gestiones conservadoras compartían una preocupación de los funcionarios provinciales por conocer la realidad, impulsando políticas tendientes a la transformación de los establecimientos carcelarios. Este informe sirvió de insumo para las autoridades políticas, y por consiguiente emprendieron reformas tomando las recomendaciones que se tradujeron en leyes y decretos. Podemos distinguir dos momentos o periodos de 
transformaciones que se ven reflejados en las medidas tendientes a seguir las propuestas de reforma. Los ritmos de estas transformaciones coinciden con las dos gobernaciones bonaerenses conservadoras de mayor estabilidad política: la de Federico Lorenzo Martínez de Hoz (1932-1935) y la de Manuel Antonio Fresco (1936-1940). Aún así el breve período de tiempo y de transición de la gobernación de Raúl Díaz -a mediados del año 35también siguió con atención las demandas exploradas por la gestión anterior. Nuestro propósito es dilucidar y describir las medidas implementadas y ponderar en que coincidieron con las recomendaciones realizadas por la comisión presidida por el Dr. Paz Anchorena para poder pensar y reflexionar sobre sus alcances y límites. Dicho de otro modo, nos interesa indagar en qué medida las recomendaciones de los expertos influyeron en la toma de las medidas gubernamentales.

No parece casual que, un mes después de haberse presentado el informe citado, se realizan los primeros movimientos de la gestión conservadora en materia penitenciaria. El Decreto N. ${ }^{\circ} 682$ del 30 de mayo de 1934 describía sucintamente las condiciones de precariedad por la cual atravesaban las cárceles provinciales, claramente aludiendo al informe de la comisión: “(...) que a este fin se han hecho prolijos estudios que los contemplan desde el punto de vista de la ciencia penal" ${ }^{5}$. Asimismo, dicho decreto destacaba la falta de un método propio del gobierno provincial para atender al aumento de la criminalidad que se veía reflejado en las estadísticas disponibles en ese momento. Frente a esta situación este decreto proponía la creación de una nueva comisión, en este caso para asesorar y proyectar reglamentaciones y el funcionamiento de los establecimientos carcelarios tal como proponía la Comisión de 1934. La nueva comisión estaba integrada por el presidente de la Suprema Corte de Justicia, Doctor César Ameghino; el Juez de Instrucción de la Capital Federal y profesor de la Facultad de Derecho de la Universidad de Buenos Aires, Dr. Eusebio Gómez; el Fiscal de Estado, Dr. Juan A. Solá; el Inspector General de Prisiones provinciales, Raúl Etcheverry; entre otros. No debe subestimarse lo importante de esta nueva comisión ya que permite entrever la preocupación que tenía el gobierno provincial sobre la problemática carcelaria y la importancia que les otorgaban a especialistas en la materia para que se ocupen de estas

${ }^{5}$ Boletín Oficial de la Gobernación de la Provincia de Buenos Aires, 1935, p. 394. 
cuestiones. La convocatoria a Eusebio Gómez, de los especialistas más reconocidos en estas problemáticas, revela esta premisa. Gómez formado en abogacía, durante las décadas del 10 y 20 se especializó en cuestiones penitenciarias y dedicó distintos escritos, a lo que debe sumarse su experiencia como Director de la Penitenciaria Nacional entre 1923 y 1928 (González 2019). Del mismo modo, el decreto establecía la inmediata construcción de tres pabellones en el penal de Sierra Chica que, como hemos visto enfatizaba el primer informe, sufría de un cuadro de superpoblación que debía ser solucionado de manera urgente. Para llevar a cabo este proyecto se invirtió la suma de 220.000 pesos $\mathrm{m} / \mathrm{n}$ que se imputaron en la Ley de presupuesto anual. Contemplando este aspecto, se encargó a la Dirección de Arquitectura la proyección y monto presupuestario para realizar la terminación definitiva del Penal de Sierra Chica y el mejoramiento de las cárceles de Mercedes, San Nicolás, Bahía Blanca y Dolores. Con la puesta en marcha de estas obras se puede advertir la interacción de las diferentes direcciones y reparticiones estatales en el momento de llevar a cabo la construcción o modificación de nuevas cárceles.

Cabe preguntarse por qué razones los planes de construcciones carcelarias no pudieron concretarse en su totalidad y algunas obras demoraron varios años en ejecutarse. Jeremías Silva (2013) señala que los magros resultados en la implementación de construcciones carcelarias bajo los gobiernos conservadores nacionales que administraban las cárceles de la Capital Federal y los Territorios Nacionales debe enmarcarse en el contexto económico desfavorable que por entonces atravesaba el país. Como es bien sabido, debido a los desajustes económicos fruto del complejo contexto internacional que afectaba a la Argentina, la gestión conservadora privilegió la obra pública orientada a modernizar la infraestructura del trasporte, riego y almacenamiento de la producción agrícola-ganadera con el fin de beneficiar a los productores rurales y favorecer la mayor eficacia de sus negocios que se encontraban más golpeados por la crisis económica del 29. Por consiguiente, se estima que el Estado sostuvo durante esos años otro tipo de prioridades que obligaron a postergar mejoras en infraestructura carcelaria (Ballent y Gorelik 2001).

Pero quizás el logro más sustancial y destacable de la gobernación Martínez de Hoz puede considerarse la reforma constitucional de ese mismo año. Para fines de noviembre, en medio de una difícil situación política que 
implicó discrepancias en el seno del partido conservador y el papel de los radicales en el recinto, se aprobó una nueva Constitución Provincial (Béjar 2004). La misma fue una reforma parcial que mantenía la estructura orgánica establecida por las constituciones de 1873 y 1889. Las modificaciones provinciales de mayor relevancia fueron: la adopción de la elección directa del gobernador y vicegobernador, la utilización del padrón nacional sobre la base del registro militar de enrolamiento y la supresión de las facultades políticas, otrora, otorgadas a las municipalidades. Por otro lado, se limitó a modificar algunos términos del preámbulo, y las cuestiones referidas a la imprenta y las contrataciones de empréstitos (Sabsay y Saba 1991). Entre estas innovaciones, el artículo 157, definía que los establecimientos carcelarios que se encontraban bajo la tutela de la Corte Suprema de Justicia provincial pasaban a manos del Poder Ejecutivo a cargo de la Inspección General de Prisiones. Esta medida, que se buscó desde el inicio de la gestión de Martínez de Hoz, se produjo cuando se encontraba a cargo el ministerio de Gobierno, Rodolfo Moreno, reconocido por impulsar múltiples proyectos legislativos de reforma carcelaria cuando se desempeñó como diputado conservador provincial durante la experiencia radical (Silva 2017). Por ello, Moreno destacaba que esta reforma:

Permitirá dar lugar a economías en el manejo de esos establecimientos, que podrán ser provistas por la Dirección General de Suministros, permitiendo tener una reglamentación uniforme dentro de los mismos y facilitará los traslados de penados una vez pronunciada las condenas. (Ministerio de Gobierno 1935, 21)

Por lo tanto, esta medida constituyó el puntapié inicial a un proceso de centralización que marcó el inicio de una autonomización de la cuestión carcelaria y la búsqueda de maximizar los esfuerzos por parte de la administración bonaerense. De esta manera, en los sucesivos meses, se tomaron medidas tendientes a atender el problema carcelario, como puede observarse en los decretos y disposiciones publicados en los Boletines Oficiales de la Provincia de Buenos Aires de la gobernación de Martínez de Hoz siguiendo las orientaciones de la comisión. Se repite de manera similar el discurso sobre las deficiencias del sistema y la inadecuación de los establecimientos y, al mismo tiempo, la gran preocupación que aquejó a la 
administración. Del mismo modo, se atendió a uno de los problemas que enfatizó la comisión presidida por Paz Anchorena en su informe: el régimen de trabajo. Sobre este aspecto, por ejemplo, en el boletín del 20 de septiembre se describe las resoluciones del Decreto 928 que explicita la relevancia de proporcionar trabajo a las personas que se encuentren recluidas. Justamente, la normativa destacaba la finalidad de los establecimientos como lugares de reforma, para alejar la imagen imperante de un sistema basado en la corrupción de las personas, por lo que buscaba garantizar un sistema de trabajo sistemático y metódico. La ordenanza definía la contratación de personas con conocimientos técnicos para que proyecten la instalación de talleres y que asesoren al gobierno sobre lo que deben proveerse para su funcionamiento eficiente.

Por otro lado, en el boletín del 22 de diciembre del mismo año mostraba los primeros resultados: estadísticas sobre la disminución de porcentaje de recluidos que permanecían ociosos, y lo relacionaba con la gran concurrencia de la mayoría de los penados a los talleres de distinta índole que se habían instalado en los establecimientos carcelarios a partir de septiembre de 1934. Los resultados para las autoridades fueron claramente beneficiosos, ya que además contaban con la formación de un fondo propio para los penados al que podían acceder una vez cumplida su condena. Asimismo, se resaltaba que los nuevos talleres les inculcaban a los presos el concepto del trabajo durante el periodo de reclusión, factor que tendría una influencia decisiva y que servía para su reinserción social. En consecuencia, lo que buscaba la gobernación era reforzar y fomentar la cultura del trabajo dentro de los talleres carcelarios y emprendió una nueva normativa dedicada a este aspecto. La nueva "Reglamentación General de Peculios y Aranceles", que se sancionó el 19 de diciembre de 1934, establecía las formas en que los penados accederían a los bienes en dinero de su propio trabajo y también conceptualizaría los beneficios a partir de categorías de conducta/competencia. Confeccionado por la Inspección General de Prisiones, que se encontraba bajo la órbita de la Dirección General de Suministros desde el año 33, esta norma abarcó a todos los establecimientos carcelarios provinciales y su objetivo además de proveer de un estímulo al recluso, permitía unificar un solo cuerpo de disposiciones en materia de peculios. Éste se depositaría en una cuenta del Banco de la Provincia llevando el registro la Contaduría General de la Provincia de manera 
centralizada. Estas iniciativas, expresan la voluntad y empeño de los funcionarios por uniformar y establecer normativas comunes a los establecimientos carcelarios, algo de lo que adolecían, tal como manifestaba el informe de 1934.

Al cabo de un tiempo y en los últimos meses de la gestión de Martínez de Hoz, a principios de 1935, la administración provincial a partir del Taller de Impresiones Oficiales imprimió 4000 folletos titulados "El problema carcelario" en el cual contenía una serie de mensajes y medidas llevadas a cabo por la gestión. Lo más destacable junto al mensaje y ciertos decretos, es que contenía el proyecto de ley para la venta de tierras fiscales pertenecientes al estado provincial y la puesta en discusión del Código Penal. Durante el periodo de elaboración, en septiembre de 1934 se realizó un censo carcelario y visitas a los establecimientos, como el presidio de Sierra Chica que debía ser atendido en torno a la cuestión edilicia. Una visita se realizó en conjunto entre la comitiva formada a partir del Informe de Paz Anchorena y que incluía además al ministro de Obra Pública; acompañados por ingenieros y fiscales estatales (Ministerio de Gobierno 1935, 17). Por lo tanto, esta designación ilumina la cooperación y el trabajo en conjunto para zanjar estas problemáticas de la mano tanto de políticos como de expertos en la materia. En este punto se valoró la labor del ingeniero Juan P. Marelli en la confección de los planos para la construcción de los pabellones en el establecimiento localizado en Olavarría. Retomando el énfasis en las construcciones y remodelaciones, la comitiva entendió que debía realizar un esfuerzo que no dañara la ya complicada situación que presentaban las arcas fiscales, por ende se puso en valor ciertas tierras públicas: funcionarios de la Dirección General de Tierras prepararon oportunamente las planillas con las propiedades a rematar y se gestionó a partir de la legislatura la autorización para que el Ejecutivo pueda llevar a cabo el proceso de licitación pública bajo una cuenta especial que no sea parte de las rentas generales. Junto a esta medida, Martínez de Hoz hizo público el estudio y el diseño de los planos para la construcción de una cárcel en la localidad de Olmos, disponiendo de los fondos que establecía la Ley 4143. Construcción que va a sufrir ciertas modificaciones hasta su inauguración en 1939 bajo la gestión de Manuel Antonio Fresco. Por último, el folleto también estipulaba llevar a cabo un proceso de reforma en torno a la cuestión reglamentaria. En ese sentido, Rodolfo Moreno, ministro de gobierno, culpaba al Código Penal 
de las falencias a la hora de decretar ciertas sentencias que justamente contraponía la verdadera misión del sistema. Del mismo modo enfatizaba el periodo de transición entre el delito y la condena como un problema que debía prestarse suma atención y que no debía pasarse por alto a partir del proyecto de ley a presentar.

En resumen, la gestión de Martínez de Hoz atendió a las recomendaciones realizadas por la comisión de expertos, en particular en lo concerniente a las reformas edilicias y en materia de trabajo para los penados. La interrupción de su gestión a manos de su propio partido, las tensiones con el ala nacionalista, que no estuvo dispuesto a seguir su directiva de encaminar el proceso a unas elecciones limpias, terminó dejando de lado los proyectos que estaban prontos a realizarse (Béjar 2005, p. 128). Lo sucedió en el cargo el vicegobernador Raúl Díaz en marzo de 1935, que poco después fue confirmado en su cargo por una intervención federal. Así, concluyó una primera etapa de reformas que buscó mejorar la administración del castigo provincial, basada en las propuestas de los expertos. Recién bajo la gobernación de Manuel Fresco en 1936 esta cuestión volvió al centro de la escena política.

En el primer aniversario de su asunción en febrero de 1937, el gobernador Manuel Antonio Fresco brindó un discurso en la legislatura provincial en el cual dedicó especial atención al problema carcelario. El estado de los establecimientos y las futuras medidas que se proyectaban impulsar concernientes a esta materia fueron la base de su disertación. El mismo enumeró los logros del primer año de gestión: la supresión del hacinamiento de detenidos en los diferentes establecimientos (Cárcel de Encausados y la Penitenciaria) de la Ciudad de La Plata, el estudio de las obras para poder finalizar con la nueva de Cárcel de Olmos y la construcción de nuevos pabellones en el Penal de Sierra Chica. Muchas de estas obras, se habían proyectado en la gestión de Martínez de Hoz y como hemos señalado tuvieron dificultades económicas para poder llevarlas a cabo. Andrés Regalsky y María Da Orden (2013) remarcan que, a pesar de que la acción de los conservadores provinciales bonaerenses durante esta década osciló entre el recorte y la adopción de medidas para atender las necesidades de la población bonaerense. Por ello, el gasto presupuestario se concentró en algunos ítems, a pesar del peso que implicó la gran deuda pública que presentaba la provincia. Sin embargo, existió entre 1937 y 1939 un leve 
período de recuperación que significó un aumento del personal estatal alcanzando los 70.000 puestos para 1938. Asimismo, el proyecto de ley sobre las tierras fiscales de la provincia para la financiación de establecimientos carcelarios quedó sin efecto. En su discurso Fresco expresaba la relevancia que adquirió la Inspección General de Prisiones de la Provincia a la que se dotó de nuevas y mayores actividades para poder afrontar las soluciones que no fueron posibles y los obstáculos que representaba motorizar cambios sustanciales en las instituciones carcelarias. Todas estas medidas fueron destacadas por la Revista Penal y Penitenciaria, órgano de difusión de la Dirección General de Institutos Penales de la Nación. La revista ocupaba un lugar central en la circulación de saberes en torno a cuestiones criminales, la reforma de los Códigos Penales, así como las experiencias y eventos carcelarios del país a nivel provincial e internacional (Silva, 2019). Por lo tanto, no solo implicó para el poder provincial un anuncio importante, sino que a nivel nacional se ponderaban las reformas provinciales de la matriz penitenciaria. Por último, Fresco definía las próximas medidas en que se iba a ocupar su gestión, focalizando en las siguientes áreas: edificación, clasificación de penados, régimen de trabajo e instrucción del personal carcelario.

En este contexto de reformas y preocupación de parte del Poder Ejecutivo Provincial, se produjo la transformación institucional más sustantiva de la administración de las prisiones bonaerense en materia penitenciaria de los años 30: la creación de la Dirección General de Establecimientos Penales (DGEP). Emulando las transformaciones burocráticas realizadas por el Estado Nacional que en 1933 conformó la Dirección General de Institutos Penales (DGIP), el gobierno de Fresco emprendió también una reorganización del sistema carcelario provincial siguiendo los mismos parámetros, y a partir de las recomendaciones cultivadas por la comisión de 1934. En este sentido, se dictó la Ley número 4555 reglamentada por el Poder Ejecutivo mediante el Decreto número 57 con fecha al 10 de junio de 1937. Por lo tanto, la centralización se hacía definitiva en el control del Ejecutivo de todas las instituciones. La DGEP pasó a asumir las tareas que poseía hasta ese momento la Inspección General de Prisiones que, por otro lado, sólo se quedó con sus funciones de fiscalización. Evidentemente tenía un sesgo centralizador para el Ejecutivo que venía a reafirmar las premisas de la reforma constitucional, en este sentido se puede conectar con las reformas 
en el organigrama policial para el mismo año bajo la gobernación de Fresco (Barrenche, 2019) Asimismo, se nombró un Consejo Asesor con funciones consultivas que sugería a la Dirección reformas y medidas. Estaba constituida bajo la presidencia del Inspector General, e integrada además por el Inspector Jefe, en compañía de los inspectores encargados de las Oficinas Técnicas. Las relaciones de la junta con el Poder Ejecutivo tendrían lugar por el claro intermedio de la Dirección General y su Director principal. El objetivo que tenía el Consejo era el de estudiar a fondo el problema carcelario y proponer reformas legislativas y medidas de orden administrativo que buscaran unificar el régimen penitenciario de la Provincia y dotarlos de una organización científica, en particular de adoptar las premisas de la criminología positivista en boga por aquellos años (Caimari 2004; Salvatore 2001; Silva 2013).

Esta nueva estructura burocrática también estuvo integrada por cuerpos técnicos y un anexo medico psicológico por el cual a partir de las clasificaciones que se realizaban se debía confeccionar la ficha criminológica. En el año 1938 finalmente se puso en práctica esta Ficha Criminológica que se basaba en la que había confeccionado Osvaldo Loudet en 1933 en tanto Director del Instituto de Criminología de la Penitenciaría Nacional y adoptada desde 1935 por la DGIP, y que serviría de modelo a diversos países de Latinoamérica (Silva 2017). Esta Ficha se proponía reunir los antecedentes de los penados clasificando la información médica, psicológica, así como los antecedentes personales y familiares y debían ser confeccionadas por médicos criminólogos.

La nueva repartición gubernamental estableció una Oficina de Edificios (en vinculación con la Dirección de Arquitectura Provincial) que estudiaban los planos y proyectos para establecimientos penales. Esta oficina era similar a la que poseía la DGIP y desarrollaba diversos proyectos al Poder Ejecutivo Nacional como el "Plan de Construcciones Carcelarias y Organización de los Establecimientos" de 1935 que buscaba proyectar con el asesoramiento de arquitectos las futuras acciones en materia penitenciaria. En efecto, con la creación de laDGEP el decreto y ley de 1937 estipulaban la inversión de 200.000 pesos $\mathrm{m} / \mathrm{n}$ para el cumplimiento de pago de sueldos, gastos y adquisición de todos los elementos que resultaban necesarios en la proyección de futuras obras carcelarias provinciales. Una de estas obras se inauguró en octubre del mismo año y consistía en cuatro pabellones en el 
Penal de Sierra Chica. Este evento que daba cuenta de la relevancia de la obra pública para los gobiernos conservadores, y en particular con la atención que la administración provincial le otorgó a ofrecer soluciones a las problemáticas carcelarias, estuvo presidida por el Gobernador Fresco. ${ }^{6}$

Para poder ver en mayor grado la obra fresquista no hay que dejar de lado la construcción definitiva de la Cárcel de Olmos. Con un costo total que se elevó a 3.300.000 de pesos $\mathrm{m} / \mathrm{n}$., el establecimiento que sugería ya el informe de Anchorena y que luego comenzaría a construirse durante la breve gobernación de Raúl Díaz en 1935, representaba una obra de infraestructura moderna que buscó ofrecer soluciones al problema de la superpoblación que se reflejaba en las estadísticas carcelarias (ya que se trasladó población carcelaria de la Cárcel de Encausados y Penitenciaria de La Plata). Esta obra de ingeniería gestionada junto a la Dirección de Arquitectura y la supervisión del Ingeniero Juan Cambiaggio se encuentra, hasta la actualidad, localizada a $15 \mathrm{~km}$. de la Ciudad de La Plata, en la localidad de Lisandro Olmos. Con un total de 35.000 metros cuadrados, un frente de 160 metros, una longitud de fondo de 190 metros de muro a muro, el establecimiento se preparaba para albergar hasta 1200 encausados. De la misma forma se instaló un servicio de cloacas, además de baños modernos con agua caliente, nuevas cocinas y "ambulancias automotores" que reemplazaban a las de tracción a sangre a la hora de transportar detenidos. Por otro lado, otra "victima" de este progreso fue el Penal de Sierra Chica, logrando la necesaria uniformidad arquitectónica: la construcción de cuatro pabellones que se sumaba a la reedificación de otros cuatro y ampliaciones en los restantes. Estas obras se destacaron por descongestionar, al menos en el corto plazo, las cárceles departamentales que sufrían de un altísimo nivel de superpoblación.

De esta manera, la convocatoria a expertos que realizó la gestión provincial a comienzos de los años 30, nutrió una vigorosa agenda con las que las gestiones conservadoras, en particular durante las gobernaciones de Martínez de Hoz y Fresco, impulsaron múltiples medidas con el fin de solucionar los déficits carcelarios provinciales. Hemos buscado mostrar la forma en que dichas medidas gubernamentales se informaron de las

${ }^{6}$ Boletín Oficial de la Gobernación de la Provincia de Buenos Aires, 1937, p. 81. 
recomendaciones que en 1934 realizó la comisión de expertos presidida por Paz Anchorena detalladas en la primera sección de este escrito.

\section{A modo de conclusión}

Este artículo se propuso recuperar los diagnósticos, propuestas y transformaciones institucionales llevadas a cabo por diferentes gestiones conservadoras de la Provincia de Buenos Aires en los años treinta. Creemos haber demostrado la preocupación que tuvieron los políticos provinciales sobre el problema carcelario bonaerense, la relación con los expertos y la centralidad de este momento en la conformación de un sistema penitenciario provincial moderno.

En el primer apartado hemos analizado el informe que el gobierno provincial encargó a una comisión de expertos en materia penitenciaria, y el detallado diagnóstico de la situación en que se encontraban las cárceles de la Provincia de Buenos Aires a comienzos de los años treinta. Los déficits que presentaba la situación provincial ofrecían un panorama carcelario marcado por la falta de las premisas que debían tener las instituciones de castigo y eran compartidas con diferentes establecimientos penitenciarios provinciales y nacionales desde comienzos del siglo XX. La falta de un régimen de trabajo, los problemas edilicios, instrucción, superpoblación y de una agencia gubernamental encargada de diseñar e implementar políticas en esta área constituyeron los reclamos y articularon las propuestas de los expertos que recorrieron las cárceles provinciales.

En el segundo apartado nos detuvimos en las transformaciones institucionales y burocráticas identificando que las reformas en materia penitenciaria se correspondieron con las gestiones provinciales de Martínez de Hoz y Fresco, momento central en la reforma carcelaria provincial, que se tradujo en las medidas tendientes a corregir los problemas más acuciantes heredados del siglo XIX. A partir del análisis de la legislación y normativa pudimos reconstruir las innovaciones que se implementaron, y ponderar en qué medida las políticas adoptaron las propuestas de los expertos. En particular, las iniciativas que atendían a problemas puntuales como la conformación de la Dirección General de Establecimientos Penales en 1937 y las diversas obras de infraestructura materializadas en estos años, revela 
el dinamismo en materia carcelaria de las gestiones conservadoras.

Justamente, los años 30 constituyeron un momento de debates, propuestas y transformaciones burocráticas en las cárceles de la Provincia de Buenos Aires que no habían merecido atención. Si bien, este caso provincial nos muestra las similitudes que se produjeron entre las innovaciones realizadas por el estado central (Silva 2013) y coincidimos con la caracterización que realizan los trabajos que indagan como la década del 30 constituyó un momento de modernización conservadora ${ }^{7}$ en el plano estatal, en nuestro caso de estudio esa transformación se nutrió de los saberes expertos (Ballent y Gorelik 2001; Caravaca 2012). Por ello nuestro trabajo buscó dialogar y ser un aporte tanto a la historiografía del castigo en la Argentina del siglo XX, como a la historia del Estado provincial.

\section{Referencias}

Ballent, Anahí. 2008. "Ingeniería y Estado: la red nacional de caminos y las obras públicas en la Argentina, 1930-1943”. História, Ciencias, SaúdeMarguinhos 15 (3): 827-847.

Ballent, Anahí y Adrian Gorelik. 2001. "País urbano o país rural: la modernización territorial y su crisis". Crisis económica, avance del estado e incertidumbre política, compilado por Alejandro Catarruzza. Buenos Aires: Sudamericana

Barreneche, Osvaldo. 2010. "Manejo metodológico de las fuentes documentales y los archivos policiales. El caso de la Policía de la Provincia de Buenos Aires". Revista electrónica de fuentes y archivos. Publicación electrónica del Centro de Estudios Históricos Prof. Carlos Segreti 1.

Barreneche, Osvaldo. 2019. De Brava a dura: Policía de la Provincia de Buenos Aires. Una historia (1930-1976). Rosario: Prohistoria Barreneche, Osvaldo y Ricardo Salvatore. 2013. El delito y el orden en

\footnotetext{
7 Siguiendo a Ballent, se entiende este como concepto como una reconfiguración técnica y tecnológica, llevada a cabo en la década del 30, a partir de aplicaciones de nuevas medidas que en términos políticos y económicos buscan mantener los centros tradicionales de la sociedad y la economía (Ballent 2008).
} 
perspectiva histórica. Rosario: Prohistoria.

Béjar, María Dolores. 2004. "La construcción del fraude y los partidos políticos en la Argentina de los años treinta". Cuadernos del CISH 1516. Disponible en: http://www.fuentesmemoria.fahce. unlp.edu.ar/art_revistas/pr.366/pr.366.pdf

- 2005. El régimen fraudulento. La política en la Provincia de Buenos Aires, 1930-1943. Buenos Aires: Siglo XXI Editores.

Belzunces, Gustavo Federico. 2020. "Transiciones hacia el encierro.

Espacios y prácticas de la prisión en la historia de la cárcel de Mercedes (1854-1882)". Revista de Historia de las Prisiones 10: 27-47.

Biernat, Carolina y Karina Ramacciotti. 2012. Politicas sociales. Entre demandas y resistencias. Argentina 1930-1970. Buenos Aires: Biblos.

Bohoslavsky, Ernesto. 2010. Un Estado con rostro humano. Buenos Aires: Prometeo Libros.

Bohoslavsky, Ernesto y Fernando Casullo. 2003. "Sobre los límites del castigo en la Argentina periférica. La cárcel de Neuquén (1904-1945)". Revista Quinto Sol 7: 37-59.

Caimari, Lila. 2001. "Ciencia y sistema penitenciario". En Academia Nacional de la Historia, Nueva Historia de la Nación Argentina, Tomo 8, págs. 471-496. Buenos Aires: Ed. Planeta.

- 2002. "Castigar civilizadamente. Rasgos de la modernización punitiva en la Argentina (1827-1930)". En Violencias, delitos y justicias en la Argentina, compilado por S Gayol y G Kessler, págs. 141-167. Buenos Aires: Manantial.

- 2004. Apenas un delincuente. Crimen, castigo y cultura en la Argentina, 1880- 1955. Buenos Aires: Editorial Siglo XXI.

Caimari, Lila y Máximo Sozzo (comps.). 2017. Historia de la cuestión criminal en América Latina. Rosario: Prohistoria

Caravaca, Jimena. 2012. "La Argentina keynesiana. Estado, política y expertos económicos en la década de 1930”. En Las prácticas del Estado: Política, sociedad y elites estatales en la Argentina del siglo XX, compilado por Mariano Ben Plotkin y Eduardo Zimmermann, págs. 6791. Buenos Aires: Edhasa.

Degregori, Daniel. 1996. "El gasto público en la Provincia de Buenos Aires, 1914-1940”. FACES 2 (2): 27-47.

Di Lisci, María Silvia y Germán Soprano (eds.). 2017. Burocracias 
estatales: problemas, enfoques y estudios de caso en la Argentina (entre fines del siglo $X I X y X X)$. Rosario: Prohistoria-EdUNLPam.

Fernández, Noelia. 2013. “'Hablando con el pueblo’ La creación de LS 11 bajo la gestión de Manuel Fresco en la provincia de Buenos Aires, 19361940”. Revista Question 1 (38).

2014. "Estado, administración y procesos políticos: El Ministerio de Obras Públicas de la Provincia de Buenos Aires entre 1917 y 1943." VIII Jornadas de Sociología de la UNLP, 3 al 5 de diciembre de 2014, Ensenada, Argentina.

Flores, Valeria. 2015. "Los inicios del sistema carcelario en el Territorio Nacional de la Pampa (1884-1930)". Revista de Historia de las Prisiones 1: 53-77.

Giacomelli, Julieta. 2018. "Religión, Estado, asistencia y sociedad: un análisis de sus relaciones a partir de las particularidades del encierro femenino en Azul (1918-1932)". Avances del Cesor 15 (19).

González Alvo. 2013. Modernizar el castigo. La construcción del régimen penitenciario en Tucumán, 1880-1916. Rosario: Prohistoria.

_ 2015. "La reforma penitenciaria en 'El Subtrópico de la República' (Tucumán, Argentina, 1881-1927)”. Revista Pilque 2: 23-39.

González, Esteban. 2019. "El Boletín de la Biblioteca Nacional de

Criminología y Ciencias Afines (1926-1929): Eusebio Gómez y la Penitenciaría Nacional de Buenos Aires)". Revista Historia y Justicia 12. León León, Marco Antonio. 2014. "Por una 'necesidad de preservación social': Cesare Lombroso y la construcción de un 'Homo Criminalis' en Chile (1880-1920)". Cuadernos de historia: 31-59

Lobato, Mirta Zaida y Juan Suriano (comps.). 2014. La sociedad del trabajo. Las instituciones laborales en la Argentina (1900-1955). Buenos Aires: Edhasa.

Milena, Luciano. 2015. La versión local del reformismo penitenciario. Córdoba, 1908- 1916. Revista de Historia de las Prisiones 1: 99-116.

Ministerio de Gobierno. 1933. "Memoria de Gobierno 1932-1933". Ministerio de Gobierno de la Provincia de Buenos Aires. La Plata: Taller de Impresiones Oficiales decretos". Ministerio de Gobierno de la Provincia de Buenos Aires. La Plata: Taller de Impresiones Oficiales. 
Ortiz Bergia, María José Ortiz. 2015. "La compleja construcción del Estado intervencionista. Lógicas políticas en la conformación de la estructura estatal en Córdoba. 1930-1955". Trabajos y Comunicaciones 42. http://www.trabajosycomunicaciones.fahce.unlp.edu.ar/article/view/Ty C2015n41a01

Palacio, Juan Manuel. 2013. Historia de la provincia de Buenos Aires: de la federalización de Buenos Aires al advenimiento del peronismo: 1880 1943. Buenos Aires: Edhasa

Palermo, Silvana A. 2006. "Elite Técnica y Estado Liberal: la Creación de una Administración Moderna en los Ferrocarriles del Estado (18701910)". Estudios Sociales 30 (1): 9-42.

https://doi.org/10.14409/es.v30i1.2569

Paz Anchorena, José María. 1934. Régimen e institutos carcelarios de la Provincia de Buenos Aires. Informe de la comisión de estudio. Ministerio de Gobierno de la Provincia de Buenos Aires. La Plata: Taller de Impresiones Oficiales.

Piazzi, C. 2011. Justicia criminal y cárceles en Rosario (Segunda mitad del siglo XIX). Rosario: Prohistoria.

Plotkin, Mariano Ben y Eduardo Zimmermann. 2012. Los saberes del Estado. Buenos Aires: Edhasa.

Regalsky, Andrés y María Da Orden. 2013. "La banca y las finanzas públicas en la Provincia de Buenos Aires (1880-1943)". En Historia de la provincia de Buenos Aires: de la federalización de Buenos Aires al advenimiento del peronismo: 1880-1943. Buenos Aires: Edhasa

Sabsay, Daniel y Roberto Saba. 1991. "El frustrado intento de reforma de la constitución de la provincia de Buenos Aires". Revista de Derecho Político 34: 331-353.

Salvatore, Ricardo. 2001. "Sobre el surgimiento del estado médico-legal en la Argentina (1890-1940)”. Estudios Sociales 20: 81-114.

Salvatore, R. y Carlos Aguirre. 1996. The Birth of the Penitentiary in Latin America. Essays on Criminology, Prison Reform, and Social Control, 18301940. Austin: UTP.

Silva, Jeremías. 2013. "El sistema penitenciario del Estado Nacional entre 1930-1943”. En El delito y el orden en perspectiva histórica, compilado por Osvaldo Barreneche y Ricardo Salvatore, págs. 227-250. Rosario: Prohistoria. 
2017. "Abandonad toda esperanza, vosotros los que entráis. Proyectos, legislación y políticas penitenciarias en Argentina (19161938)". En Historia de la cuestión criminal en América Latina, compilado por Lila Caimari y Máximo Sozzo, págs. 317-356. Rosario: Prohistoria Ediciones.

. 2019. "La Revista Penal y Penitenciaria: órgano de difusión de una burocracia moderna (1936-1946)". Revista Historia y Justicia 12.

Ternavasio, Marcelo (dir.). 2013. Historia de la provincia de Buenos Aires. De la organización federal a la federalización de Buenos Aires, 18211880. Buenos Aires-Gonnet: Edhasa-UNIPE.

Yangilevich, Melina Silva. 2017. "Vínculos complejos: cárceles, estado y sociedad en la provincia de Buenos Aires (Argentina) durante la segunda mitad del siglo XIX". Claves. Revista de Historia 3 (4). 